\title{
Left ventricular volume: an optimal parameter to detect systolic dysfunction on prospectively triggered 64-multidetector row computed tomography: another step towards reducing radiation exposure
}

\author{
Mohit Gupta • Yalcin Hacioglu • Jigar Kadakia • \\ Naser Ahmadi $\cdot$ Yanlin Gao $\cdot$ Song S. Mao • \\ Matthew J. Budoff
}

Received: 7 April 2010/Accepted: 23 October 2010/Published online: 10 November 2010

(C) The Author(s) 2010. This article is published with open access at Springerlink.com

\begin{abstract}
In this study, we define the correlation between LV volumes (both LV end-diastolic volume [LVEDV] and LV end-systolic volume [LVESV]) and ejection fraction (EF) on 64 slice multi-detector computed tomography (MDCT). We also determine the accuracy of all the LV volume (LVV) parameters to detect LV systolic dysfunction (LVSD) and investigate the feasibility of using LVV as a surrogate of LVSD on prospectively gated imaging to prevent the radiation exposure of retrospective imaging. 568 patients undergoing 64-detector MDCT were divided into 2 groups: Group 1-subjects without any heart disease and LVEF $\geq 50 \%$; and Group 2-patients with coronary artery disease and LVEF $<50 \%$ (defined as LVSD). The LVV (LV cavity only) and Total LV volume (cavity + LV mass) at end-systole and end-diastole (LVESV, Total LVESV, LVEDV and Total LVEDV) were measured. The upper limit values (mean $+2 \mathrm{SD}$ ) of all LVV parameters in Group 1 were used as the reference criterion to diagnose LVSD in Group 2. An exponential correlation was found between LVEF and all the LVV parameters. The specificity to detect LVSD in Group 2 was $>90 \%$ and the sensitivity was $88.9,83.3,61.3$
\end{abstract}

M. Gupta $(\bowtie) \cdot$ Y. Hacioglu · J. Kadakia ·

N. Ahmadi - Y. Gao · S. S. Mao - M. J. Budoff

Division of Cardiology, Los Angeles Biomedical

Research Institute at Harbor UCLA, 1124 W Carson St.,

Torrance, CA, USA

e-mail: mohit_gupta13@yahoo.com and $74.9 \%$ by using LVESV, Total LVESV, LVEDV and Total LVEDV, respectively. Systolic and diastolic LV volumes had a high correlation with LVEF and a high accuracy to detect LVSD. Thus, on prospectively triggered imaging, ventricular volumes can predict patients with reduced LVEF, and appropriate referrals can be made.

Keywords Computed tomography angiography . Multi-detector row computed tomography - Ejection fraction - End diastolic volume - End systolic volume $\cdot$ Radiation reduction $\cdot$ Prospective imaging

\section{Introduction}

Left ventricular (LV) systolic function and LV volumes are the fundamental parameters for diagnosis, disease stratification, prognosis estimation, and therapeutic management of ischemic and nonischemic cardiomyopathy. LV volume (LVV) and myocardial mass are independent predictors of morbidity and mortality among patients with coronary artery disease (CAD), and global LV function is considered the strongest determinant of heart failure and death due to myocardial infarction. Due to its prognostic relevance, accurate and reproducible determination of LV volumetric and functional parameters is a major clinical requirement for diagnosis and risk stratification of patients with suspected or documented heart disease [1-4]. 
Global LV function and chamber volumes measured with Multi-detector computed tomography (MDCT) have been found to be in good agreement with echocardiography, cine ventriculography, single photon emission computed tomography (SPECT) and cardiac magnetic resonance imaging (CMRI) [5-9]. ECG-gated MDCT of the heart can provide retrospective quantitative information on LV volume changes through all the phases of cardiac cycle ( $\mathrm{R}-\mathrm{R}$ interval) and consecutive information on global LV function [9]. However, with the recently increased concern for radiation exposure, new MDCT protocols scanner use prospectively acquired ECG gated image acquisition to obtain images at certain phases of cardiac cycle with least coronary motion. These protocols do not allow assessment of functional data which involves measurement of ejection fraction $[E F]$ requiring end-systolic and end-diastolic phases.

LV end-diastolic volume (LVEDV) and LV endsystolic volume (LVESV) have been validated to diagnose and follow LV volumetric and functional abnormalities on echocardiography, CMRI and LV angiogram [10-15]. In this study, we aim to define the correlation between LV volumes (LVEDV and LVESV) and ejection fraction on 64 slice MDCT. We also determine the accuracy of all the LVV parameters to detect LV systolic dysfunction (LVSD) and investigate the feasibility of using LVV as a surrogate of LVSD on prospectively gated image acquisition. This would result in assessment of LV function along with interpretation of coronary arteries with prospective imaging, without exposing the patient to the radiation dose of retrospective imaging.

\section{Methods}

Study population

This study consisted of 568 consecutive patients who underwent 64 slice MDCT at our center (Los Angeles Biomedical Research Institute at Harbor-UCLA) from September 2006 to June 2009. The various indications for the study included: chest pain, shortness of breath, abnormal or equivocal stress test, cardiomyopathy, congestive heart failure, or syncope. Patients were excluded if found to have an irregular heart rate, allergy to contrast medium or impaired renal function. The study was approved by the local Institutional Review Board of our hospital.

The patients were divided into two groups: Group 1 included subjects without any coronary artery disease, hypertension, lung disease or any wall motion abnormalities having a normal LVEF $\geq 50 \%$ ( $\mathrm{n}=281$ ); Group 2 included patients with coronary artery disease and a LVEF of $<50 \% \quad(n=287)$ (Table 1).

\section{Patient preparation}

The patients were prepared in usual manner as described in earlier studies. In brief, patients received explanation about the procedure and informed consent was obtained. Upon arrival to our center, if the patient's base line heart rate [HR] was more than $80 \mathrm{bpm}, 100 \mathrm{mg}$ of atenolol was given orally and the patient was asked to wait for $1 \mathrm{~h}$. Alternatively, if the $\mathrm{HR}<80 \mathrm{bpm}$ the patient was placed on the scanner without any delay. On the scanner, if the HR was $>60 \mathrm{bpm}$, intravenous (IV) metoprolol was

Table 1 Demographics and Classifications of Studied Individuals

\begin{tabular}{|c|c|c|c|c|c|c|c|}
\hline Gender & $\mathrm{N}$ & Age (year) & Weight (kg) & Height $(\mathrm{cm})$ & $\operatorname{BSA}\left(\mathrm{m}^{2}\right)$ & Heart rate $(\mathrm{BPM})$ & LVEF $(\%)$ \\
\hline \multicolumn{8}{|c|}{ Group $1(\mathrm{n}=281)$} \\
\hline Female & 115 & 57.1 & 71.0 & 161.5 & 1.74 & 57.8 & 65.3 \\
\hline Male & 166 & 54.3 & 89.4 & 177.0 & 2.06 & 55.8 & 63.8 \\
\hline \multicolumn{8}{|c|}{ Group $2(n=287)$} \\
\hline Female & 75 & 63.1 & 161.9 & 62.9 & 1.75 & 58.7 & 36.1 \\
\hline Male & 212 & 61.4 & 190.8 & 69.7 & 2.03 & 57.6 & 34.4 \\
\hline
\end{tabular}

Group 1 = patients without any heart disease and a normal LV Ejection Fraction (LVEF) $\geq 50 \%$

Group 2 = patients with coronary artery disease and a LVEF of $<50 \%$ 
administered in doses of $5 \mathrm{mg}$ every $1 \mathrm{~min}$ until the HR was brought below $60 \mathrm{bpm}$ or a total IV dose of $40 \mathrm{mg}$ was given or the systolic blood pressure (SBP) dropped to $<90 \mathrm{~mm} \mathrm{Hg}$. Intravenous diltiazem was also used to complement the beta blocker if needed. Three phase injection using a Dual Barrel Injector with contrast/saline mix was administered intravenously as follows: $5 \mathrm{ml} / \mathrm{s}$ for $12 \mathrm{~s}(60 \mathrm{ml}$ contrast), then $5 \mathrm{ml} / \mathrm{s}$ for $10 \mathrm{~s}(50-20 \mathrm{ml}$ contrast plus $30 \mathrm{ml}$ saline) and finally $5 \mathrm{ml} / \mathrm{s}$ for $4 \mathrm{~s}$ (20 $\mathrm{ml}$ of saline), injection protocols were varied based upon patient characteristics (length of heart, heart rate, body habitus).

\section{MDCT study protocol}

All the Coronary Computed Tomography Angiography (CCTA) studies were completed with 64 MDCT (LightSpeed VCT, General Electric Medical Systems, Milwaukee, WI). The technique of the cardiac retrospective overlapping helical acquisition was completed using a 0.35 -s scan gantry rotation speed and a $64 \times 0.625 \mathrm{~mm}$ collimation. The reconstruction field of view was small $(12-17 \mathrm{~cm}$, mean $15 \mathrm{~cm})$ with a voxel size of $0.35 \times 0.35 \times 0.6 \mathrm{~mm}^{3}$. An energy setting of $120 \mathrm{kVp}$ was used for patients weighing $\geq 85 \mathrm{~kg}$. For patients who were $<85 \mathrm{~kg}$, an energy setting of $100 \mathrm{kVp}$ was selected. The tube current time product (mAs) was 200-770, determined individually based on the body habitus and weight. The total scan time was about 5-6 s. The target HR was 50-60 beats/min, achieved using oral and intravenous beta blockers as described above. After scan completion, multi-phase reconstruction of the CCTA scans was performed, with reconstructed images from 5 to $95 \%$ phases by $10 \%$ increments [16]. All CCTA images were transferred to a reading center for 3-dimensional image analysis on Advantage Windows (AW) 4.4 Workstation (General Electric Medical Systems, Milwaukee, WI).

LVV and LV ejection fraction measurement with 64 slice MDCT

All LV segmentations were measured by using 64 slice MDCT retrospective multi-phase images. A global estimation of wall motion was initially done. Then the axial images with minimum and maximum diameter were chosen for manual segmentation analysis to calculate the LV end-systolic and end- diastolic diameters, respectively. The segmentation analysis included delineation of LV endocardial boundary at end-systole (to calculate LVESV including only the LV cavity) and epicardial boundary at both end-diastole and end-systole (to calculate Total LVEDV and Total LVESV including the LV cavity and the LV mass). The endocardial tracing was only done in end-systole to decrease the CT partial volume effect since the trabeculae and papillary muscle could be separated from the cavity significantly in these images. The LVEDV was computed by the following formula (Fig. 1) [17, 18]:

\section{Stroke volume $(\mathrm{SV})=$ Total LVEDV$$
\text { - Total LVESV; and LVEDV }=\mathrm{SV}+\mathrm{LVESV}
$$

In each study, 10-14 axial slice levels were traced, while the remaining slices were automatically traced by the workstation, and visually confirmed by the reader. The volume measured by the endocardial and epicardial tracing lines corresponded to LV cavity volume (LVV) and Total LV volume (inclusive of LVV + LV mass), respectively. Volumes were calculated in two workstations using modified Simpson's method, in which the sum of cross-sectional areas was multiplied by the sum of slice thicknesses. The LVEF was then computed by the formula $[17,18]: \mathrm{LVEF}=\mathrm{SV} / \mathrm{LVEDV} \times 100 \%($ Fig. 1$)$.

\section{Statistical analysis}

The data was analyzed using SPSS 15 statistical software. Categorical variables were analyzed using Chi-square test and Continuous variables using student $t$ test. Mean values and Standard Deviations (SD) of all the LVV measurements were calculated. The correlation between LV volumes and gender and body surface area (BSA) was calculated by using the Pearson's method. Considering the significant linear association between LV volumes, and gender and body size ( $\mathrm{r}>0.3, P<0.05$ for all), the gender and BSA specific LV volumes were calculated for all measurements. All subsequent analyses were performed using $\mathrm{LV}$ volume indexes. $T$ test was used to test the difference between genders and the two groups. For all analyses, a criterion for statistical significance was set at a 2 -tailed $P$ value $<0.05$.

The correlation of LVEF with all the LVV parameters was determined and correlation coefficient 


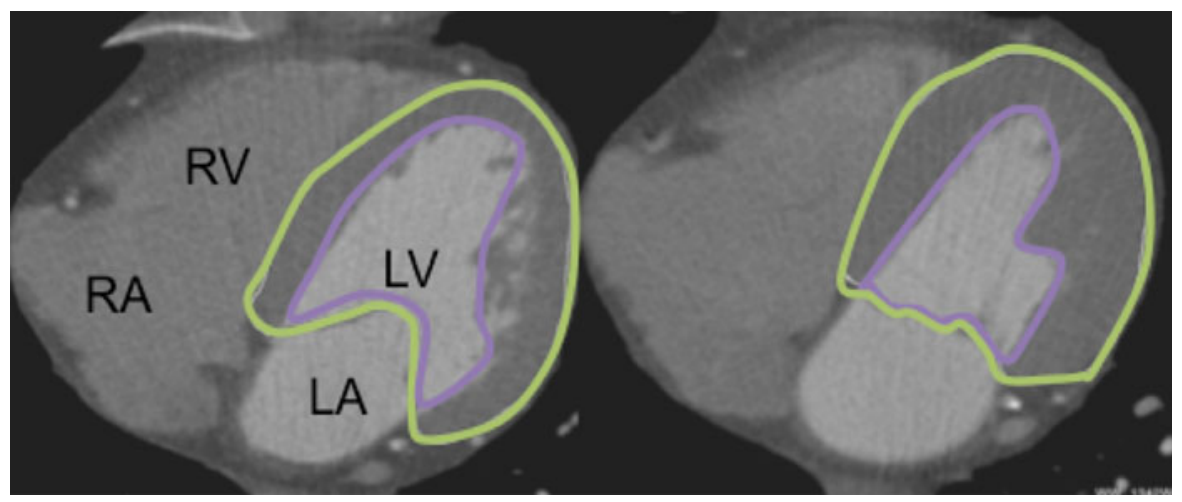

Fig. 1 LVV and LVEF measurement by 64 MDCT. Right and left panels represent systolic and diastolic phases, respectively. The LV cavity volume at end-systole (LVESV) was traced manually, marked as the inner purple line. The total LV volume at end-diastole and systole (Total LVEDV and Total LVESV including the LV mass and cavity) were traced and

$\left(\mathrm{r}^{2}\right)$ calculated. The upper limit values (2 SD above the mean) of all LVV parameters in Group 1 were then used as the reference criteria to diagnose LVSD in Group 2. LVSD was defined as LV ejection fraction $<50 \%$, measured by MDCT. The accuracy of these reference criteria (95th percentile) to diagnose LVSD in Group 2 was then computed.

\section{Results}

The mean age for this patient cohort was 61.7 years and $38 \%$ were females. The mean values for LVESV, Total LVESV, LVEDV and Total LVEDV as computed in Group 1 were $19.3 \pm 6.9,88.1 \pm 14.9,54.3 \pm 13.2$, and $123.2 \pm 20.19 \mathrm{ml} / \mathrm{m}^{2}$ and the upper limit values calculated as above ( $2 \mathrm{SD}$ above the mean) were 33 , 118,81 and $163 \mathrm{ml} / \mathrm{m}^{2}$, respectively (Table 2).

Using the upper limits as the reference values, the specificity to detect LV systolic dysfunction in Group 2 was $>90 \%$ and the sensitivity was $88.9,83.3,61.3$ and $74.9 \%$ by LVESV, Total LVESV, LVEDV and marked as the outer green line. LVV was traced and Total LVV was calculated automatically by multiplication of summed area to the thickness of slice. The LVEF was computed manually through: LV stroke volume $(\mathrm{SV})=$ Total LVEDV - Total LVESV,$\quad$ LVEF $=\mathrm{SV} /($ LVESV $+\mathrm{SV}) \times 100 \% . \quad L A \quad$ left atrium; $L V$ left ventricle; $R A$ right atrium; $R V$ right ventricle

Total LVEDV parameters, respectively. Area under the curve (AUC) was also computed to determine the accuracy of all the LVV parameters to detect LVSD. (Fig. 2; Table 3).

An exponential correlation was found to exist between the LVEF and the LVESV $\left(r^{2}=0.74\right)$, Total LVESV $\left(r^{2}=0.64\right), \operatorname{LVEDV}\left(r^{2}=0.48\right)$ and Total LVEDV $\left(r^{2}=0.49\right)$. Thus, all the LVV parameters had a good correlation with LVEF and a high sensitivity and specificity to detect LV systolic dysfunction. (Fig. 3; Table 3).

\section{Discussion}

Traditional acquisition protocol for CCTA has been to obtain all phases of the cardiac cycle (R-R interval) allowing calculation of EF simultaneously with plaque burden. Recently increased concern for radiation exposure has led to a shift towards prospectively triggered acquisition protocols for CCTA imaging [19]. These protocols target specific
Table 2 Profile of mean values and upper limits for all the LV volume parameters

\begin{tabular}{lccc}
\hline Model & $\begin{array}{l}\text { Mean } \pm \text { SD Group 1 } \\
\left(\mathrm{ml} / \mathrm{m}^{2}\right)\end{array}$ & $\begin{array}{l}\text { Upper limit of normal } \\
\left(\mathrm{ml} / \mathrm{m}^{2}\right)(\text { mean }+2 \mathrm{SD})\end{array}$ & $\begin{array}{l}\text { Mean } \pm \text { SD Group 2 } \\
\left(\mathrm{ml} / \mathrm{m}^{2}\right)\end{array}$ \\
\hline LVESV & $19.3 \pm 6.9$ & 33 & $64.2 \pm 42.6$ \\
Total LVESV & $88.1 \pm 14.9$ & 118 & $162.7 \pm 60.0$ \\
LVEDV & $54.3 \pm 13.2$ & 81 & $92.4 \pm 46.6$ \\
Total LVEDV & $123.2 \pm 20.2$ & 163 & $192.6 \pm 65.2$ \\
\hline
\end{tabular}




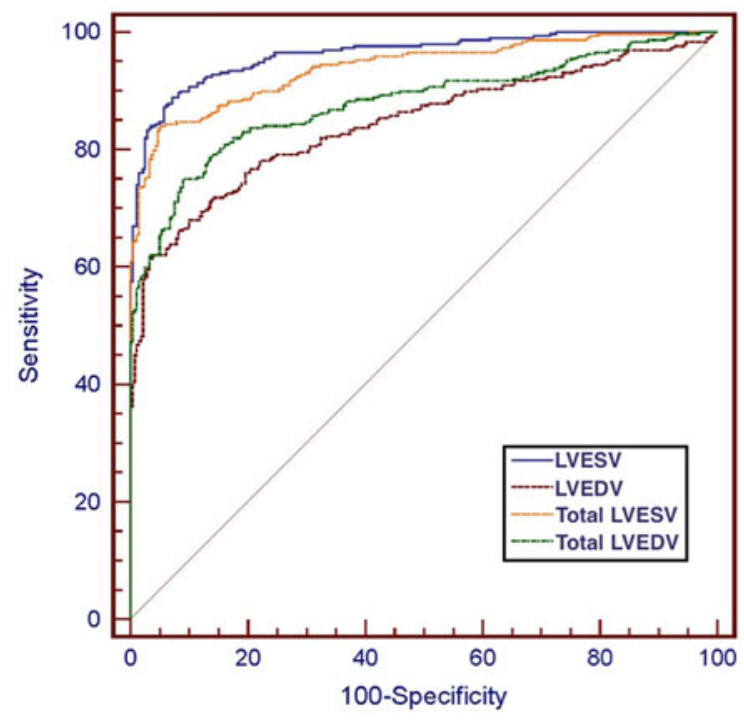

Fig. 2 Receiver-operating characteristic (ROC) curve for accuracy of LV volume parameters to detect LV systolic dysfunction

phases of the $\mathrm{R}-\mathrm{R}$ interval that have been shown to produce the best coronary image quality but do not allow assessment of functional data which involves measurement of ejection fraction requiring endsystolic and end-diastolic phases. Gerber et al. provided a comprehensive scientific statement on radiation doses from the American Heart Association and estimated the doses for retrospective CCTA at 15 milli Seivert $(\mathrm{mSv})$ without tube modulation, $9 \mathrm{mSv}$ with dose modulation and $3 \mathrm{mSv}$ for prospectively acquired studies [20]. Minimizing radiation exposure through ECG triggered X-ray tube activation is a step towards making CCTA safer.

In our study we reviewed the CCTA's performed by retrospectively triggered 64 slice MDCT of 568 patients to determine the correlation between LV volumes and ejection fraction on MDCT. Our goal was to investigate the feasibility of using LVV as a surrogate of LVSD on prospectively gated image acquisition. We found that all the LVV parameters had a significant correlation with LVEF and were found to be sensitive indicators of LVSD. As more cardiac CT studies are obtained in prospective (single phase) mode to reduce radiation exposures, clinicians can use increased ventricular volume as a marker of reduced LVEF and consider further evaluation, increasing the yield of prospective studies without incremental radiation exposure.

An interesting observation in our study was that amongst all the LVV parameters, LVESV was the most sensitive $(88.9 \%)$, specific $(92.9 \%)$ and accurate (AUC-0.96) in detecting LVSD. LVESV has been shown to have important implications in literature. Increase in LVESV after exercise independently predicts mortality in patients with CAD and provides useful complementary information for risk stratification [21]. It is also an indicator of remodeling after myocardial necrosis from infarction [22, 23], having a greater predictive value than EF or LVED with the latter two not adding any prognostic value [3, 24]. The association of resting LVESV to mortality and incident heart failure is also known [25]. During stress or exercise, EF may not adequately reflect contractile reserve since the EF is affected by loading conditions and is largely driven by the degree of LV dilatation. In comparison, LV volume may be a more sensitive indicator of ventricular function, especially at the limits of contractile reserve where contractility is unable to match the physiologic increase in preload and after load during exercise [21]. LV volumes are easily measurable and reproducible, less sensitive to cardiac loading and vary greatly in response to changes in contractility.

LV dilation results from infarct expansion [26] due to slippage of the necrotic fibers on one another as the infarct stretches during systole [27]. Infarct expansion occurs between 3 days and 2 weeks after

Table 3 Accuracy of LV volume parameters to diagnose LV systolic dysfunction

\begin{tabular}{lllllll}
\hline Model & AUC $( \pm$ SE) & Sensitivity $(\%)$ & Specificity $(\%)$ & $P$ value & $95 \%$ CI & $\mathrm{r}^{2}$ with EF \\
\hline LVESV & $0.96(0.008)$ & 88.9 & 92.9 & 0.0001 & $0.94-0.98$ & 0.74 \\
Total LVESV & $0.94(0.01)$ & 83.3 & 95.4 & 0.0001 & $0.92-0.96$ & 0.64 \\
LVEDV & $0.84(0.01)$ & 61.3 & 96.8 & 0.0001 & $0.81-0.87$ & 0.48 \\
Total LVEDV & $0.88(0.01)$ & 74.9 & 91.1 & 0.0001 & $0.85-0.91$ & 0.49 \\
\hline
\end{tabular}

$A U C$ area under the receiver-operating curve, $S E$ standard error 

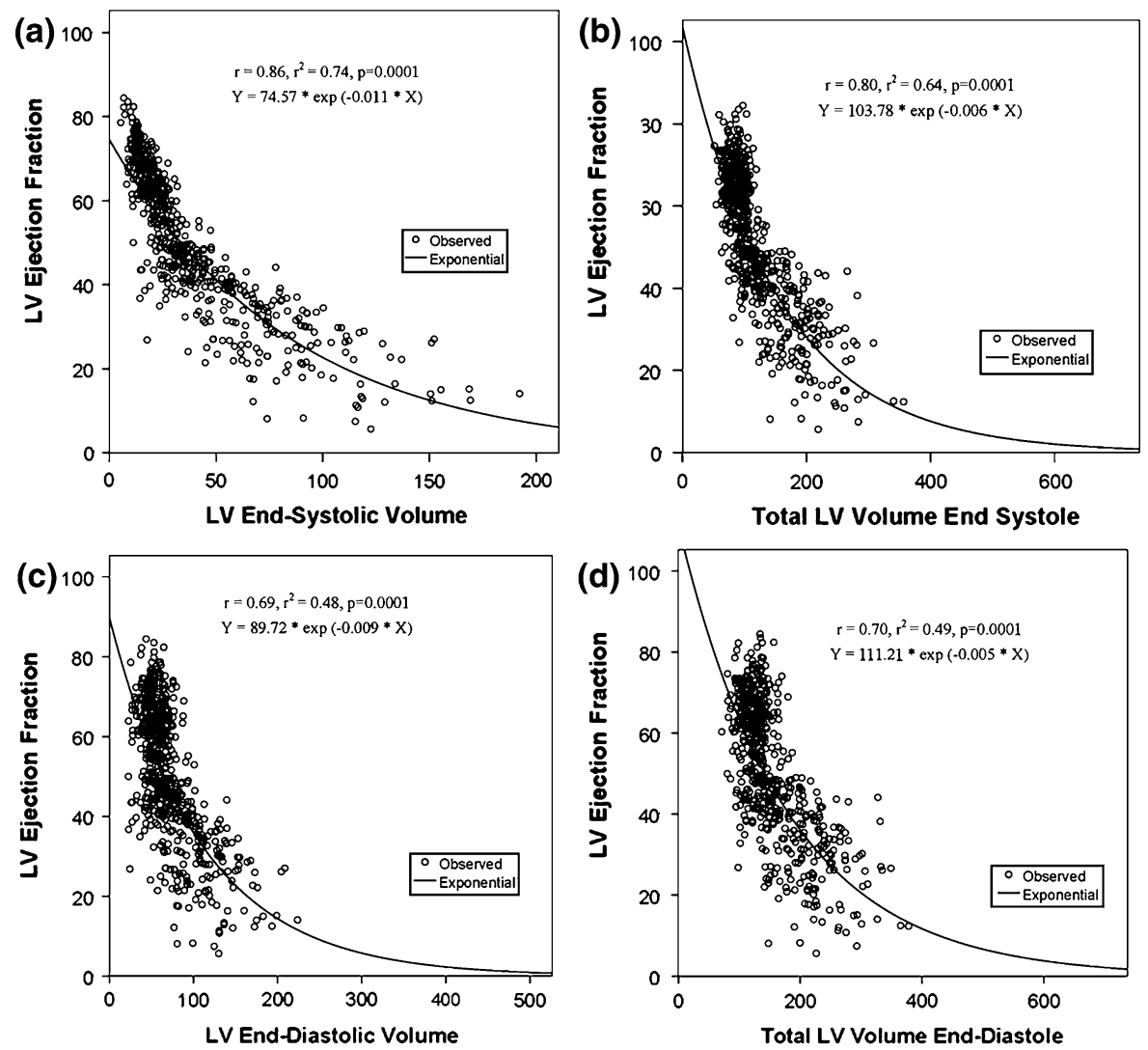

Fig. 3 a Correlation between LV ejection fraction and LV end-systolic volume. b Correlation between LV ejection fraction and Total LV end-systolic volume. c Correlation

infarction [28], and patients showing expansion by 10-21 days after transmural infarction may continue to have expansion over a period of 6-30 months [29]. Infarct expansion would increase LV systolic and diastolic volumes with resultant increase in wall stress, which in turn may act as a stimulus to cardiac hypertrophy [30]. Thus, LV dilation after infarction has been identified as a major risk factor for subsequent cardiac death [3].

To date, ventricular dimensions have most commonly been assessed by echocardiography or in patients undergoing invasive catheterization, by left ventriculography based on mono or biplane projections. However, both measurements rely on geometric assumptions about ventricular structure [9]. MDCT can accurately differentiate the endocardial and epicardial boundaries with high spatial resolution and provide detailed information of cardiac structures that allows precise measurement of the chamber between LV ejection fraction and LV end-diastolic volume. d Correlation between LV ejection fraction and Total LV enddiastolic volume

volume without assumptions regarding geometry [17]. In the evaluation of cardiac function and chamber volumes, MDCT with a temporal resolution of 125-250 ms and high spatial resolution has been shown to be a promising alternative to echocardiography, biplanar cine ventriculography, SPECT and CMRI [9].

In the present study, we demonstrated all the LVV parameters had a high correlation with LVEF and a high sensitivity to detect LVSD. Thus, MDCTmeasured LVV could be used as a complementary or confirmatory parameter to LVEF in early diagnosis of LVSD. LV volumes may also serve as a surrogate for LVEF whenever its estimation is technically difficult or impossible due to various reasons. This may increase the confidence of clinicians in diagnosis and early management of LVSD and prevent further progression of disease. As prospectively triggered scans are unable to measure LVSD [31], using 
ventricular volumes as a marker of LVSD on these scans can lead to a significant radiation dose reduction in comparison to getting a retrospective ECGgated CT scan for the same information.

In a prospective triggered scan, depending on patient's heart rate and its regularity, the single acquired phase is usually neither the LVEDV nor the LVESV. Additionally, in our study, LVESV showed to be the most accurate parameter in detecting LVSD, but LVESV is normally not determined in prospective triggered CCTA. LVEDV, which matches most closely to the mid-diastole LV volume measured in prospective triggered CCTA [31], however, showed only a fair sensitivity to detect LVSD (61\%). Hence based on our study, the transfer of the results of retrospective gated scans to prospectively triggered CCTA is limited but gives a foundation to future studies to further validate this concept.

\section{Limitations}

Our study has some limitations. Although LV volumes measured with MDCT have shown good correlation with the values obtained by CMRI in various studies, currently CMRI is the non-invasive diagnostic standard of reference for determination of LV volumes demonstrating a high diagnostic accuracy and low inter and intra-observer variability. With a lower temporal resolution than CMRI, MDCT is more susceptible to motion artifacts, especially during systole and atrial contraction. Because of this, systolic images obtained in patients with a higher heart rate, are of lower quality and may impair delineation of endocardial contours [9]. Also, the measurements for LVEF in our study were made on MDCT. Having a reference standard for LVSD in terms of echocardiography or CMRI would have further strengthened our findings.

Secondly, not all the patients can be scanned using prospective ECG triggered CT image acquisition, a common example being patients with atrial fibrillation. The maximum dose saving is achieved using the shortest acquisition window, which does not allow any flexibility in post acquisition phase adjustment. This can potentially result in sub-optimal image quality and compromise the diagnostic value of the exam.
The measurement of LV volumes as described in our study can be tedious and cumbersome to do in daily clinical practice. However, this has been done for research purpose for this validation study. In routine practice, physicians can use the automatic software programs available on every CCTA reading workstations to compute LV volumes. For e.g: LV volumes can be generated using the 'Auto Ejection Fraction' program on Advantage Windows (AW) workstation.

\section{Conclusion}

The results of our study demonstrate that all the LV volume parameters on CT allow detection of LVSD. Since MDCT-derived LV volumes are easy to measure and highly reproducible, they can be used for early detection of LVSD as a surrogate or in addition to LVEF to facilitate early management and prevent further progression of congestive heart failure. Since most CT studies will be utilizing prospective triggering and thus unable to measure LVSD, measure of LV volume should provide important information to the interpreting physician as to the presence of possible LV dysfunction, without exposing the patient to the radiation dose of retrospective imaging.

Open Access This article is distributed under the terms of the Creative Commons Attribution Noncommercial License which permits any noncommercial use, distribution, and reproduction in any medium, provided the original author(s) and source are credited.

\section{References}

1. Hammermeister KE, DeRouen TA, Dodge HT (1979) Variables predictive of survival in patients with coronary disease: selection by univariate and multivariate analyses from the clinical, electrocardiographic, exercise, arteriographic, and quantitative angiographic evaluations. Circulation 59:421-430

2. Shah PK, Maddahi J, Staniloff HM et al (1986) Variable spectrum and prognostic implications of left and right ventricular ejection fraction in patients with and without clinical heart failure after acute myocardial infarction. Am J Cardiol 58:387-393

3. White HD, Norris RM, Brown MA, Brandt PW, Whitlock RM, Wild CJ (1987) Left ventricular end-systolic volume as the major determination of survival after recovery from myocardial infarction. Circulation 76:44-51 
4. Juergens KU, Seifarth H, Range F et al (2008) Automated threshold-based 3D segmentation versus short-axis planimetry for assessment of global left ventricular function with dual-source MDCT. Am J Roentgenol 190(2):308-314

5. Schlosser T, Pagonidis K, Herborn CU et al (2005) Assessment of left ventricular parameters using 16-MDCT and new software for endocardial and epicardial border delineation. Am J Roentgenol 184(3):765-773

6. Dewey M, Müller M, Eddicks S et al (2006) Evaluation of global and regional left ventricular function with 16-slice computed tomography, biplane cineventriculography, and two-dimensional transthoracic echocardiography: comparison with magnetic resonance imaging. J Am Coll Cardiol 48(10):2034-2044

7. Sarwar A, Shapiro MD, Nasir K et al (2009) Evaluating global and regional left ventricular function in patients with reperfused acute myocardial infarction by 64-slice multidetector CT: a comparison to magnetic resonance imaging. J Cardiovasc Comput Tomogr 3(3):170-177

8. Agarwal PP, Patel S, Corbett J et al (2009) Left ventricular functional analysis with 16- and 64-row multidetector computed tomography: comparison with gated singlephoton emission computed tomography. J Comput Assist Tomogr 33(1):8-14

9. Orakzai SH, Orakzai RH, Nasir K, Budoff MJ (2006) Assessment of cardiac function using multidetector row computed tomography. J Comput Assist Tomogr 30(4):555-563

10. Hershberger RE, Ni H, Crispell KA (1999) Familial dilated cardiomyopathy: echocardiographic diagnostic criteria for classification of family members as affected. J Card Fail 5(3):203-212

11. Haji SA, Ulusoy RE, Patel DA et al (2006) Predictors of left ventricular dilatation in young adults (from the Bogalusa Heart Study). Am J Cardiol 98(9):1234-1237

12. Lee SH, Yoon SB, Cho JR, Choi S, Jung JH, Lee N (2008) The effects of different beta-blockers on left-ventricular volume and function after primary coronary stenting in acute myocardial infarction. Angiology 59(6):676-681

13. Mann-Rouillard V, Fishbein MC, Naqvi TZ, DonMichael TA, Siegel RJ (1999) Frequency of echocardiographic improvement in left ventricular cavity size and contractility in idiopathic-dilated cardiomyopathy. Am J Cardiol 83(1):131-133, A9-10

14. Theisen D, Sandner TA, Bauner K et al (2009) Unsupervised fully automated inline analysis of global left ventricular function in CINE MR imaging. Invest Radiol 44(8):463-468

15. Staal EM, de Heer M, Jukema JW et al (2003) End-diastolic and end-systolic volume from the left ventricular angiogram: how accurate is visual frame selection? Comparison between visual and semi-automated comnputerassisted analysis. Int J Cardiovasc Imaging 19(4):259-266

16. Budoff MJ, Dowe D, Jollis JG et al (2008) Diagnostic performance of 64-multidetector row coronary computed tomographic angiography for evaluation of coronary artery stenosis in individuals without known coronary artery disease: results from the prospective multicenter ACCURACY (Assessment by Coronary Computed Tomographic Angiography of Individuals Undergoing Invasive Coronary Angiography) trial. J Am Coll Cardiol 52(21):1724-1732
17. Budoff MJ, Ahmadi N, Sarraf G et al (2009) Determination of left ventricular mass on cardiac computed tomographic angiography. Acad Radiol 16(6):726-732

18. Mao S, Takasu J, Child J, Carson S, Oudiz R, Budoff MJ (2003) Comparison of LV mass and volume measurements derived from electron beam tomography using cine imaging and angiographic imaging. Int $\mathbf{J}$ Cardiovasc Imaging 19(5):439-445

19. Gopal A, Mao SS, Karlsberg D, Young E, Waggoner J, Ahmadi N, Pal RS, Leal J, Karlsberg RP, Budoff MJ (2009) Radiation reduction with prospective ECG-triggering acquisition using 64-multidetector computed tomographic angiography. Int J Cardiovasc Imaging 25(4):405-416

20. Gerber TC, Carr JJ, Arai AE, Dixon RL, Ferrari VA, Gomes AS, Heller GV, McCollough CH, McNitt-Gray MF, Mettler FA, Mieres JH, Morin RL, Yester MV (2009) Ionizing radiation in cardiac imaging: a science advisory from the American Heart Association Committee on Cardiac Imaging of the Council on Clinical Cardiology and Committee on Cardiovascular Imaging and Intervention of the Council on Cardiovascular Radiology and Intervention. Circulation 119(7):1056-1065

21. Turakhia MP, McManus DD, Whooley MA, Schiller NB (2009) Increase in end-systolic volume after exercise independently predicts mortality in patients with coronary heart disease: data from the Heart and Soul Study. Eur Heart J 30(20):2478-2484

22. Pfeffer MA, Braunwald E (1990) Ventricular remodeling after myocardial infarction. Experimental observations and clinical implications. Circulation 81(4):1161-1172

23. Gaudron P, Eilles C, Kugler I, Ertl G (1993) Progressive left ventricular dysfunction and remodeling after myocardial infarction. Potential mechanisms and early predictors. Circulation 87(3):755-763

24. Burns RJ, Gibbons RJ, Yi Q et al (2002) The relationships of left ventricular ejection fraction, end-systolic volume index and infarct size to six-month mortality after hospital discharge following myocardial infarction treated by thrombolysis. J Am Coll Cardiol 39(1):30-36

25. Vasan RS, Larson MG, Benjamin EJ, Evans JC, Levy D (1997) Left ventricular dilatation and the risk of congestive heart failure in people without myocardial infarction. N Engl J Med 336(19):1350-1355

26. Hutchins GM, Bulkley BH (1978) Infarct expansion versus extension: two different complications of acute myocardial infarction. Am J Cardiol 41(7):1127-1132

27. Weisman HF, Bush DE, Mannisi JA, Weisfeldt ML, Healy B (1988) Cellular mechanisms of myocardial infarct expansion. Circulation 78(1):186-201

28. Eaton LW, Weiss JL, Bulkley BH, Garrison JB, Weisfeldt ML (1979) Regional cardiac dilatation after acute myocardial infarction: recognition by two-dimensional echocardiography. N Engl J Med 300(2):57-62

29. Erlebacher JA, Weiss JL, Eaton LW, Kallman C, Weisfeldt ML, Bulkley BH (1982) Late effects of acute infarct dilation on heart size: a two dimensional echocardiographic study. Am J Cardiol 49(5):1120-1126

30. Grossman W, Jones D, McLaurin LP (1975) Wall stress and patterns of hypertrophy in the human left ventricle. J Clin Invest 56(1):56-64 
31. Isma'eel H, Hamirani YS, Mehrinfar R, Mao S, Ahmadi N, Larijani V, Nair S, Budoff MJ (2009) Optimal phase for coronary interpretations and correlation of ejection fraction using late-diastole and end-diastole imaging in cardiac computed tomography angiography: implications for prospective triggering. Int J Cardiovasc Imaging 25(7):739-749 